\title{
Activisme sur Internet et discours stratégiques autour de la propriété intellectuelle
}

Yana Breindl, François Briatte*

Le développement des technologies de l'information et de la communication, et en premier lieu la généralisation de l'accès à Internet, a contribué à transformer l'activisme politique. La littérature consacrée à ce phénomène récent fait clairement ressortir deux évolutions. D'une part, certains événements politiques auparavant délimités dans l'espace tendent à changer d'échelle et prennent une dimension transnationale, voire supranationalel. D'autre part, les moyens disponibles pour mener certaines activités politiques, en particulier de type contestataire ou protestataire, ont été affectés à différents degrés par la mise à disposition de nouveaux répertoires d'action collective sur Internet². Le «net-activisme », tel que présenté dans ce numéro, invite ainsi à s'interroger sur les formes de l'action collective dans les sociétés où l'accès aux nouvelles technologies s'est rapidement démocratisé au cours de la dernière décennie ${ }^{3}$.

*Yana Breindl est doctorante à l'Université Libre de Belgique (ybreindl@ulb.ac.be); François Briatte est doctorant à l'Institut d'Études Politiques de Grenoble (f.briatte@ed.ac.uk). Nous remercions les personnes ayant accepté de nous renseigner dans le cadre de nos terrains de recherche respectifs, ainsi que les organisateurs du numéro pour leur patience et leur aide.

1 Pour une introduction à la globalisation des enjeux politiques sous l'effet du développement des technologies de l'information et de la communication, cf. D. della Porta, Transnational Protest and Global Activism, Rowman \& Littlefield Publishers, 2004 ; E. Hanson, The Information Revolution and World Politics, Lanham, Rowman \& Littlefield, 2008.

${ }^{2}$ Un répertoire d'action collective désigne un moyen d'action sélectionné de manière consciente par les acteurs sur la base de leur expérience passée et de leurs stratégies ; cf. C. Tilly, La France conteste de 1600 à nos jours, Paris, Fayard, 1986.

${ }^{3}$ On trouvera des éléments d'analyse et de théorie sur ce point dans O. Blondeau et L. Allard, L'activisme sur Internet, entre défection et expérimentation, Paris, Amsterdam, 2007 ; cf. également W. Bennett, « Communicating Global Activism », Information, Communication \& Society, vol. 6, n²2, 2003, p. 143-168; M. Diani, "Social Movement Networks, Virtual and Real », Information, Communication $\mathcal{E}$ Society, vol. 3, n³, 2000, p. 386-401 ; et les textes réunis par M. McCaughey, M. Ayers (dir.), Cyberactivism: Online Activism in Theory and Practice, Londres, Routledge, 2003. 
À partir des deux terrains d'enquête présentés dans ce texte, il s'agira de comprendre, dans quelle mesure la mobilisation et la coordination de l'action collective ont pu évoluer sous l'influence de la communication électronique (computer-mediated communication). Se pose également la question de la continuité entre moyens d'action, c'est-à-dire du rapport entretenu par les publics actifs sur Internet aux répertoires d'action collective traditionnels du militantisme politique.

\section{Une approche par les mobilisations législatives autour du logiciel libre}

Les processus législatifs parlementaires, aux niveaux national et européen, constituent des moments d'étude privilégiés de l'activisme centré sur la propriété intellectuelle ${ }^{4}$. À partir de deux terrains d'enquête distincts construits sur la base d'entretiens non directifs avec des activistes issus du milieu du logiciel libre et de données recueillies en ligne, le texte suivant retrace succinctement l'organisation de deux mouvements d'opposition: le premier à la directive européenne sur la brevetabilité des inventions mis en œuvre par ordinateur (dite «brevets logiciels », YB), le deuxième à la loi française relative au droit d'auteur et aux droits voisins dans la société de l'information (loi «DADVSI», FB) d'autre part. Ces initiatives juridiques ont été interprétées par leurs opposants comme des tentatives de recherche de rente et de domination du secteur du logiciel libre par des acteurs industriels privés, suscitant en retour un fort mouvement de protestation parmi les partisans du logiciel libre, regroupés dans des collectifs ad hoc très actifs sur Internet.

L'approche retenue dans ce texte consiste dès lors à saisir précisément les formes de ces mobilisations menées par des groupements d'acteurs formés en majeure partie par des développeurs et des utilisateurs de logiciels libres. Les normes et les valeurs de cette catégorie d'acteur expliquent en partie ses interventions dans les processus législatif et politique relatifs à la propriété intellectuelle. En effet, la philosophie et les mécanismes de participation sous-jacents au développement du logiciel libre sont en grande partie antinomiques aux principes qui guident le développement du régime actuel de propriété intellectuelle, fondé sur la restriction de l'accès aux ressources autrefois publiques et sur

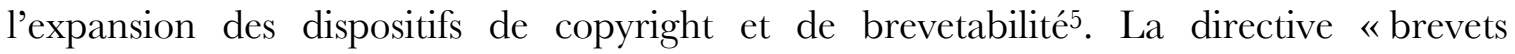

\footnotetext{
${ }^{4}$ Pour une vue d'ensemble de ces enjeux, cf. le numéro spécial de Terminal sur la propriété intellectuelle et le numérique $\left(\mathrm{n}^{\circ} 102,2008\right)$.

${ }^{5}$ Pour une présentation critique du régime actuel de propriété intellectuelle, cf. J. Boyle, «The Second Enclosure Movement and the Construction of the Public Domain ", Law \& Contemporary Problems, vol. 66, $n^{\circ} 1-2$, p. 33-74; pour une mise en perspective tout aussi critique mais plus détaillée, cf. C. May et S. Sell, 
logiciels » et la loi «DADVSI » ont ainsi eu pour effet de matérialiser cette antinomie et d'inciter à l'action collective lors de l'examen et du vote des textes.

Une caractéristique récurrente des questions de propriété intellectuelle réside dans leur grande technicité, qui nécessite de faire appel à des notions de droit privé ${ }^{6}$, à des notions d'économie appliquée aux brevets et aux biens culturels ${ }^{7}$, et dans les cas étudiés, à des notions d'informatique. L'important capital informationnel et culturel nécessaire à la compréhension des enjeux liés au droit d'auteur et aux brevets constitue en ce sens une barrière à l'entrée très efficace à l'égard des nouveaux entrants potentiels (c'est-à-dire des personnes souhaitant se saisir du sujet et en débattre en connaissance de cause). Au niveau des pouvoirs publics, le manque de temps, la classe d'âge et les origines professionnelles du personnel politique rendent d'autant plus difficile le débat sur les questions de propriété intellectuelle liées aux nouvelles technologies. En conséquence, les activistes qui souhaitaient déclencher un débat politique contradictoire autour de l'évolution des droits de propriété intellectuelle ont agi principalement sur l'information disponible aux décideurs politiques.

Intellectual Property Rights : A Critical History, Boulder, Lynne Rienner Publishers, 2005. Sur les soubassements idéologiques du logiciel libre, cf. http://www.gnu.org/philosophy/free-sw.html et les textes de Richard Stallman; sur ses principes de fonctionnement, cf.J. Feller et al. (dir.), Perspectives on Free and Open Source Software, Cambridge, MIT Press, 2005, ainsi que le numéro spécial de Terminal sur les logiciels libres ( $\mathrm{n}^{\circ} 80$ 81, 1999).

${ }^{6}$ Il s'agit principalement mais non exclusivement du droit de la propriété littéraire et artistique et du droit de la propriété industrielle, réunis en France dans le Code de la propriété intellectuelle. Les formes de ce droit varient d'un pays à l'autre et un volume très importants d'accords internationaux - ratifiés et signés à l'Organisation Mondiale de la Propriété Intellectuelle, à l'Organisation Mondiale du Commerce et au sein de l'Union Européenne - existe en matière de propriété intellectuelle; pour une vue d'ensemble, cf. H. MacQueen et al. (dir.), Contemporary Intellectual Property : Law and Policy, Oxford, Oxford University Press, 2007.

7 Pour une introduction à la brevetabilité logicielle, cf. B. Caillaud, «La propriété intellectuelle sur les logiciels », in J. Tirole (dir.), Propriété intellectuelle, rapport du Conseil d’Analyse Économique, n41, Paris, La Documentation française, 2003, p. 113-171. Pour une introduction aux enjeux du droit d'auteur, cf. F. Benhamou et J. Farchy, Droit d'auteur et copyright, Paris, La Découverte, 2007 ; parmi les publications les plus récentes, cf. également O. Bomsel, Gratuit! Du déploiement de l'économie numérique, Paris, Gallimard, 2007 ; X. Greffe et N. Sonnac, Culture web, Paris, Dalloz, 2008. 
Ge travail d'information possède deux traits saillants. Premièrement, les discours stratégiques tenus par les activistes montrent que la défense du logiciel libre nécessite de faire appel à des arguments situés à la marge des discours dominants sur la propriété intellectuelle, loin de la vision légale et économique dont les acteurs politiques tirent leur «expérience doxique » de l'enjeu ${ }^{8}$. Ce travail de rapatriement des idées reléguées à la périphérie des interprétations courantes en matière de propriété intellectuelle a exigé des activistes un véritable travail de recadrage du sujet. Deuxièmement, cet effort de déplacement cognitif s'est appuyé sur des actions collectives menées en partie sur Internet. Leur stratégie de surveillance du travail législatif, couplée à la circulation libre d'une information claire sur le contenu des textes en discussion, a permis aux groupes d'activistes d'exercer une forme de pression informationnelle sur la classe politique et sur certaines franges de l'opinion publique, à défaut de pouvoir se prévaloir des autres registres classiquement mobilisés par les groupes d'intérêt.

\section{La mobilisation contre la directive « brevets logiciels "}

La mobilisation contre les «brevets logiciels » s'est cristallisé autour du projet de directive soumis le 20 février 2002 par la Commission européenne afin d'harmoniser les législations nationales sur la brevetabilité des inventions mises en œuvre par ordinateur. Dès le début du processus législatif, des développeurs et utilisateurs de logiciels libres et open source dénoncent la remise en cause de la non-brevetabilité des programmes d'ordinateurs telle qu'établie depuis 1973 par la Convention de Munich et forment un réseau d'opposition à l'extension de la brevetabilité aux logiciels. Ce mouvement, qui se caractérise par sa compétence technique collective et la faible expérience politique de la majorité de ses membres, se structure autour de la Foundation for a Free Information

${ }^{8}$ Les croyances, expériences et attitudes doxiques sont celles qui s’imposent comme naturellement et objectivement exactes aux agents, et qui n'appellent par conséquent aucun contrepoint. Dans l'analyse qu'en fournit Pierre Bourdieu (notamment dans La distinction. Critique sociale du jugement, Éditions de Minuit, Paris, 1979), les sociétés reposent sur une «structure doxique » qui assure la reproduction de certaines normes et de certains comportements sociaux; dans le cas de la propriété intellectuelle, les croyances doxiques reposent en grande partie sur le droit, sa jurisprudence et les motifs économiques invoqués en défense de l'expansion du droit d'auteur ou de la brevetabilité ; l'entérinement de ces croyances par les médias, et notamment par la "presse intellectuelle supérieure », contribuent à former la structure doxique évoquée par Bourdieu. Le droit de la propriété intellectuelle se prête tout particulièrement à cette caractérisation, dans la mesure où l'histoire de sa conception établit clairement son statut de « reflet direct des rapports de force existants, où s'expriment les déterminations économiques, et en particulier les intérêts des dominants » (P. Bourdieu, « La force du droit », Actes de la Recherche en Sciences Sociales, n64, 1986, p. 3). 
Infrastructure (FFII) et de l'« Alliance Eurolinux » qui interviennent tout au long de la procédure de codécision ${ }^{9}$. Il réunit une coalition supranationale d'activistes présents dans de nombreux États membres, ce qui lui permet de s'impliquer dans les débats nationaux en y exerçant des pressions afin d'influencer indirectement le processus européen.

Les moyens de communication électroniques ont joué un rôle crucial dans la constitution et le fonctionnement de ce mouvement d'opposition, dans la mesure où, selon l'un des leaders de cette coalition, "pour un groupe de pression composé d'activistes provenant de toute l'UE voire au-delà, il n'y a pas d'alternative à l'utilisation extrême de la communication électronique. Il n’y a pas de possibilité de se rencontrer physiquement, en tout cas pas fréquemment $»^{10}$. Internet remplit tout d'abord une fonction de partage des informations au sein du groupe. Les listes et forums de discussion tels que ceux du réseau Linux ont largement contribué à la politisation de la communauté. C'est par ces interactions, initialement centrées sur des débats techniques, que les informations relatives aux brevets logiciels - illustrées par l'exemple des Etats-Unis, où les brevets logiciels sont légaux - ont circulé.

En plus de son effet géographiquement désenclavant, qui permet à des groupements locaux de se mobiliser sur un enjeu global, Internet permet également d'accéder à des informations autrefois réservées à une élite administrative. Les différentes institutions européennes disposent de sites Internet, et des plateformes telles que EUR-Lex procurent un accès indifférencié aux réglementations européennes. Obtenir des informations précises, sur les dépôts d'amendements par exemple, nécessite toutefois une certaine maîtrise de l'environnement institutionnel complexe de l'Union européenne. En conséquence, les activistes ont également réorganisé l'information disponible. En mettant à profit leurs compétences techniques, ils s'approprient le débat en recourant à des services qui leur permettent de sauvegarder et de disséquer les textes communautaires de manière collaborative $^{11}$. Le monitoring des parties adverses est également une ressource dans ce

${ }^{9}$ L'adoption d'une directive européenne nécessite un accord entre le Parlement européen et le Conseil de l'Union Européenne (UE). De 2003 à 2005, le projet de directive sera amendé à plusieurs reprises et finalement rejeté par un vote en deuxième lecture au Parlement européen le 6 juillet 2005.

10 F. Mueller, No Lobbyist As Such: The War over Software Patents in the European Union, Starnberg, SWM Software-Marketing, 2006, p. 47.

11 Le traitement de l'information fait ainsi intervenir l'utilisation de serveurs FTP et de wikis dédiés à la cause (le site de la FFII est également une base d'informations conséquente sur le sujet). Il est intéressant de 
processus informationnel, les activistes s'appliquant à documenter publiquement les travers du lobbying traditionnel. La surveillance de l'activité des acteurs industriels a pu constituer une arme de choix pour les détracteurs de la brevetabilité des logiciels, notamment lors de la dénonciation de la campagne faussement indépendante « Campaign for Creativity», financée par de grands groupes industriels ${ }^{12}$. Enfin, de par le volume d'informations qu'ils génèrent grâce à leur stratégie communicationnelle, les collectifs anti-brevets modifient également la perception de la directive par les acteurs médiatiques, parvenant à devenir un point de passage obligé dans la production de l'information sur ce dossier particulier ${ }^{13}$.

Outre son rôle dans le traitement de l'information par les mouvements actifs sur la directive, Internet facilite également leur mobilisation par le biais de sites et de pétitions en ligne lancés dans le courant de la campagne. Celle initiée par l'Alliance EuroLinux le 15 juin 2000, rapidement signée par plus de 100000 personnes, eut l'effet d'asseoir la crédibilité de l'association comme acteur représentant des intérêts divers. En parallèle, les personnes abonnées aux listes de diffusion furent invitées à contacter leurs représentants nationaux et/ou européens et à participer à des manifestations de rue organisées dans différentes villes européennes — en premier lieu au siège du Parlement européen à Strasbourg, entraînant le report du vote parlementaire en première lecture. Internet fit également office de site d'action en tant que tel lorsqu'environ 1500 sites Internet de la communauté Linux et open source fermèrent en signe de protestation contre la directive à deux reprises, en août 2003 et avril 2004. L'organisation de ces actions, marquantes de par leur charge symbolique, ne vise alors pas uniquement à attirer l'attention des médias

constater que l'accès à l'information via Internet est à ce point essentiel au mouvement que certaines revendications sont émises en ce sens dès le début du processus législatif: en mars 2001, l'Alliance EuroLinux avait ainsi interpellé la Commission européenne sur le manque de transparence pendant la période de consultation; cf. http://lwn.net/2001/0315/a/eurolinux-consult.php3.

12 Cf. http://kwiki.ffii.org/CampaignForGreativityEn. Cette initiative remportera d'ailleurs un prix du «plus mauvais lobbying européen en 2005 ».

${ }^{13}$ En témoigne notamment cette déclaration sur le site de la FFII : «Beaucoup de personnes au sein des institutions bruxelloises, surtout au Parlement européen, lisent régulièrement notre fil d’information sur les brevets logiciels. C'est devenu la source des couvertures les plus exactes sur le sujet [...] Nous sommes devenus de facto la principale agence de presse sur les informations concernant les brevets logiciels en Europe »; cf. leur document «Organising the Economic Majority in 2005 », http://old.ffii.org/proj/plan/. 
de masse. Des sites d'information spécialisés comme Slashdot ou Heise constituent des cibles plus accessibles dont le lectorat est également plus réceptif aux problèmes soulevés. Cette stratégie ne supprime toutefois pas la nécessité d’interpeller les supports médiatiques conventionnels et, par ce truchement, les structures institutionnelles classiques du système politique.

Sur cet aspect crucial de l'action collective, l'utilisation d'Internet a offert des canaux de communication essentiels à la diffusion des idées à l'intérieur du mouvement tout en renforçant le travail collaboratif et en encourageant le développement de nouvelles actions de terrain. Le mouvement d'opposition aux brevets logiciels a ainsi pu profiter d'une certaine crédibilité, et l'absence de hiérarchie et de stratégie claire autorisait une grande flexibilité dans leurs actions. Toutefois, les activistes ne se sont jamais délivrés de leur position d'outsider dans le processus législatif. Ils ne pouvaient ni compter sur un accès privilégié aux plateformes médiatiques ou à des " personnes ressources ${ }^{14}$ ni disposer d'une information suffisamment complète pour empêcher la multiplication de rumeurs et d'incertitudes au sein même du mouvement. L'absence de structures établies conduit également à certaines incohérences et à des tensions entre les différents acteurs impliqués ${ }^{15}$. Cet écueil logistique fait écho à ses difficultés de financement. Basé en grande partie sur le bénévolat, le manque de ressources propres fait souvent obstacle à la poursuite des tâches liées au traitement de l'information et à la présence dans les institutions communautaires ${ }^{16}$, faisant courir le risque de ne pas pouvoir assurer une campagne effective dans les moments cruciaux du travail d'opposition. Ce fut notamment le cas lors du vote parlementaire en seconde lecture, dont le résultat doit au moins autant aux mobilisations collectives autour de la procédure législative qu'à la politique interne

\footnotetext{
${ }^{14}$ La coalition a pu néanmoins bénéficier du soutien de quelques personnalités et courants politiques au sein des différentes institutions communautaires, l'organisation de conférences ayant essentiellement pour objectif de rencontrer les acteurs impliqués sur le projet de directive.

15 Après le rejet de la directive, le mouvement s'est d'ailleurs rapidement désagrégé ; trois ans plus tard, seules quelques personnes très impliquées sont encore actives, mais les problèmes de concertation se sont amplifiés.

16 La FFII observait ainsi en 2005 que « souvent, à des moments importants, le travail [de suivi de l'information] n'est pas fait. Le nombre d'activistes compétents reste limité. Plusieurs personnes seraient disponibles pour travailler à temps plein ou au moins à mi-temps »; cf. «Organising the Economic Majority in $2005 »$, op. cit.
} 
des institutions communautaires ${ }^{17}$.

Ces limitations ne doivent toutefois pas occulter les succès du mouvement d'opposition aux brevets logiciels dans le développement de répertoires d'action collective en ligne, ainsi que son écho politique dans le milieu du logiciel libre. Le «net-activisme » déployé au niveau supranational par des organisations comme la FFII a en effet fourni à des groupes comme EUCD.info une première expérience européenne de résistance à l'extension législative du régime de propriété intellectuelle. Cet apprentissage s'est effectivement prolongé dans le contexte national français, comme l'illustre l'épisode politique et législatif de la loi «DADVSI ».

\section{La mobilisation contre la loi « DADVSI »}

Dans le milieu du logiciel libre français, l'activisme autour de la loi relative au droit d'auteur et aux droits voisins dans la société de l'information (DADVSI) fait suite à la découverte d'un avantprojet de loi rédigé par une commission spéciale du Conseil Supérieur de la Propriété Littéraire et Artistique. Les dispositions du texte, notamment celles portant sur le contournement des mesures techniques de protection, entérinent les revendications de l'industrie culturelle ${ }^{18}$ et s'opposent frontalement au principe d'interopérabilité qui soustend, dans de nombreux cas, le développement de logiciels libres ${ }^{19}$. Créé en novembre

${ }^{17}$ F. Mueller, No Lobbyist As Such, op. cit., p. 332 et passim.

18 Il s'agit principalement des sociétés françaises assurant la représentation des ayants droits, appuyées ponctuellement par des firmes transnationales comme Apple, Microsoft, Virgin et Sony. Ces acteurs sectoriels sont présents de manière récurrente dans l'élaboration des marchés numériques de biens culturels (cf., par exemple, J.-S. Beuscart, La construction du marché de la musique en ligne, thèse de sociologie, École Normale Supérieure de Cachan, 2006).

${ }^{19}$ Les dispositions légales dites d'anti-contournement (anti-circumvention) visent à assurer une protection légale aux mesures techniques de protection, plus connues sous le nom de Digital Rights Management (DRM), introduites par les producteurs afin de contrôler l'usage des fichiers qu'ils distribuent. La combinaison de ces dispositifs a suscité une vive opposition entre ayants droits et utilisateurs dès leur introduction dans des traités internationaux en 1996, puis aux Etats-Unis en 2000 et en Europe par la directive 2001/29/CE (dite directive «INFOSOC » ou «EUCD », pour European Union Copyright Directive), dont DADVSI est une loi de transposition. Les activistes du logiciel libre revendiquent par ailleurs une opposition « ontologique » entre DRM et logiciel libre ; cf. le texte http://eucd.info/index.php?2004/01/28/41-intervention-ddm. 
2002 par la branche française de la Free Software Foundation, le collectif «EUCD.info »20 développe alors un discours d'opposition aux évolutions du droit de la propriété intellectuelle prévues par la loi «DADVSI».

Afin de peser sur l'activité parlementaire lors de l'examen du texte, les participants d'EUCD.info sont amenés à se former et à s'informer suivant une triple contrainte. Il leur faut d'abord accumuler le capital culturel juridique requis pour s'impliquer activement dans le processus législatif. Cette initiation au droit passera en partie par le recours à des experts extérieurs, mais se fera également de manière autodidacte ${ }^{21}$, dans le temps qui sépare la découverte de l'avant-projet de loi et le début de l'examen parlementaire, fin décembre 2005. Il leur faut ensuite disposer d'un contre-argumentaire résistant aux lignes de clivages politiques telles qu'elles se matérialisent à l'Assemblée nationale. La nécessité de ne pas recouper les antagonismes partisans s'impose afin de sensibiliser des parlementaires dans l'opposition, où certains soutiens pouvaient être considérés comme acquis (par le biais de collaborations passées notamment), mais également dans la majorité, ce qui pose une difficulté toute particulière dans un régime où l'autorité gouvernementale prévaut nettement sur celle de sa majorité, et sur le contrôle parlementaire en général. Il leur faut enfin rédiger des amendements protégeant certains principes fondamentaux, au premier rang desquels l'interopérabilité, afin de présenter leur action comme une force de défiance constructive.

Cette stratégie d'intervention se fonde sur un réquisitoire à charge contre le projet de loi en faisant intervenir ses conséquences sur l'industrie du logiciel libre, les libertés individuelles mais aussi la sécurité intérieure et la compétitivité économique de la France $^{22}$. Elle constitue la clé de voûte du travail d'EUCD.info auprès de la classe politique, par contacts directs et envois postaux, et auprès du public, notamment via leur

\footnotetext{
20 Notre travail d'enquête s'est concentré sur ce collectif, dont le site (http://eucd.info/) est encore partiellement accessible ou reconstituable via un service d'archivage (http://archive.org/). EUCD.info a coexisté avec d'autres groupes, notamment l'association AFUL, le collectif StopDRM et la Ligue Odebi, dont l'influence sur le travail parlementaire n'est pas aussi marquée.

${ }^{21}$ Sur l'apprentissage au contact des nouvelles technologies, cf. F. Bonvin, J.-P. Faguer, « Une génération d'autodidactes ", Actes de la Recherche en Sciences Sociales, n¹34, 2000, p. 76-83.

22 Pour une vue d'ensemble de l'argumentaire déployé par EUCD.info en vue des débats parlementaires, cf. http://www.eucd.info/documents/dossier-eucd-vl.pdf et en particulier la représentation graphique.
} 
site Internet, sur la mailing-list de la Free Software Foundation, et sur le forum «Libertés Numériques » d'un site de logiciels libres très fréquenté, Framasoft. Ils s'appliquent également à concurrencer les acteurs gouvernementaux ou industriels dans la production de l'information dominante, en publiant une partie de leur argumentaire dans une revue spécialisée sur la sécurité internationale. En définitive, la transversalité de l'argumentaire sur l'interopérabilité et son couplage à d'autres considérations économiques introduites par les associations de consommateurs vont soumettre le projet de loi à un examen parlementaire catastrophique pour le ministère de la Culture et de la Communication²3. En l'état, la loi «DADVSI » est aujourd'hui inopérante, ce que confirme la relance dès $2007 \mathrm{du}$ processus législatif autour du projet de loi «Création et Internet», ou «ADOPI $»^{24}$.

La communication électronique, fortement instituée dans le milieu du logiciel libre ${ }^{25}$, se prête également à d'autres initiatives visant à soutenir collectivement le travail argumentaire et discursif accompli par EUCD.info. Ces soutiens recouvrent de très nombreuses situations, allant de la mobilisation d'individus chargés de finaliser le publipostage des courriers aux députés à l'agrégation par voie pétitionnaire d'intérêts très divers : des groupes idéologiquement très hétérogènes et distincts de par la structure de leurs intérêts propres convergent ainsi sur la signature de la pétition en ligne « NON au projet de loi DADVSI ${ }^{26}$. Certains moyens de pression «physiques », comme l'assistance

23 L'examen se terminera sur le vote par les deux chambres d'un texte illisible dont les principales dispositions seront finalement censurées par une décision surprenante du Conseil Constitutionnel, saisi par l'oppositionNe pouvant inclure de chronologie complète ou de commentaire détaillé du texte par manque de place, on se reportera au récit de D. Lahary, «Les bibliothèques et la loi DADVSI. Survivre dans un débat fracassant », Bulletin des bibliothèques de France, vol. 51, n5, p. 18-25, et au rapport de synthèse d'André Lucas dans le numéro spécial de la revue juridique spécialisée Propriétés Intellectuelles (n²5, 2007); cf. également l'entrée Wikipedia http://fr.wikipedia.org/wiki/Histoire_de_la_loi_DADVSI.

${ }^{24}$ Les acteurs d'EUCD.info, réinvestis dans le collectif «La Quadrature du Net » sont également très présents sur ce projet de loi en cours d'examen, auquel ils appliquent un traitement similaire. La production politique de ce projet de loi se construit d'ailleurs sur deux niveaux, national et européen; cf. http://www.laquadrature.net/fr/olivennes et le site afférant.

${ }^{25}$ D. Demazière, F. Horn, M. Zune, « La dynamique de développement des communautés du logiciel libre. Conditions d'émergence et régulations des tensions », Terminal, n97-98, p. 71-84.

${ }^{26}$ W. Bennett, «Communicating Global Activism », op. cit. La pétition d'EUCD.info, lancée le 2 décembre 2005, dépassera les 150000 signatures et produira un écho médiatique dans la presse écrite et télévisuelle. 
aux débats parlementaires ou les appels au siège d'un parti politique, ont également été amplifiés par une mobilisation préalable en ligne. D’autres stratégies s'appliquent intégralement en ligne, comme le suivi des débats parlementaires à partir du site Internet de l'Assemblée Nationale. La traduction de l'information vers l'anglais permet enfin de partager l'actualité du mouvement avec d'autres groupes d'activistes et de bénéficier d'une couverture médiatique internationale.

Les activistes du logiciel libre (dont ceux d'EUCD.info) s'essaieront également à des moyens d'action plus conventionnels, dans le but d'en exploiter la charge symbolique. C'est le cas notamment de la «Marche pour les Libertés Numériques » du 7 mai 2006, qui se termine par un dépôt de gerbes de fleurs devant le Ministère de la Culture et un lâcher de ballons blancs. D'autres groupes interviendront également dans l'espace public par le biais de «flash-mobs». Ces mouvements de faible ampleur suggèrent que la communication électronique a joué un rôle crucial dans la constitution d'un répertoire d'action collective efficace dans l'opposition à la loi «DADVSI». De même, il faut réinterroger la remarque de Peter Dahlgren selon laquelle, «avec le net, la politique extra-parlementaire devient plus facile à pratiquer ${ }^{27}$. Le cas d'étude abordé ici montre qu'Internet peut également être mis au service d'une action de pression tout à fait conventionnelle, centrée sur les acteurs législatifs dans leur activité routinière (interventions lors des débats, soumission d'amendements, interpellation du ministre, etc. $)^{28}$. Les moyens employés par les «net-activistes » du logiciel libre autour de l'examen de la loi «DADVSI» renvoient plutôt au recours raisonné et symbolique de la communication électronique, à des fins de mobilisation d'une plus large fraction du corps

Son succès considérable, accentué par l'échec de sa remise au Premier ministre, surprendra totalement ses auteurs. L'analyse statistique des signatures réalisée par EUCD.info confirme que la pétition a réuni des publics proches (ingénieurs et informaticiens constituent environ 25\% des signatures individuelles étudiées), mais également des intérêts disparates dans le corps enseignant et les professions intellectuelles supérieures ; cf. http://eucd.info/documents/Etude_statistique_petition_EUCD-INFO_20060303_v1.pdf.

${ }^{27}$ P. Dahlgren, «L'espace public et l'internet », Réseaux, n¹00, p. 177.

${ }^{28}$ Peter Dahlgren envisage d'ailleurs cette possibilité lorsqu'il remarque que les « configurations actuelles du pouvoir » résisteront probablement à l'extension d'Internet dans le corps social, et que son effet s'en tiendra principalement à «nourrir des sphères publiques plus nombreuses et approfondir ainsi - et élargir l'espace public dominant » (ibid., p. 177-178). 
social mais surtout à des fins stratégiques, liées à la diffusion d'un argumentaire critique sur les évolutions du droit de la propriété intellectuelle.

\section{Conclusion : les dimensions symboliques du « net-activisme "}

La constitution d'un discours d'opposition sur la propriété intellectuelle et sa diffusion par Internet témoignent du rôle joué par la communication électronique dans le renouvellement des répertoires d'action collective disponibles à des mouvements comme celui du logiciel libre. Elles montrent également les limites de ce répertoire, ainsi que ses connexions avec d'autres formes de protestation organisée, comme l'activité pétitionnaire. Elles permettent enfin d'émettre l'hypothèse que, sans l'utilisation de ces nouveaux répertoires, il n'aurait pas été possible d'organiser si efficacement le recadrage cognitif recherché par les acteurs contestant la vision dominante de la propriété intellectuelle, ce qui suggère que le recours au «net-activisme » n'est pas uniquement instrumental — il ne s'agit pas seulement de faire baisser les coûts de coopération.

En effet, dans les deux cas étudiés, et contrairement aux remarques générales formulées par Mario Diani29, les pratiques des activistes ayant recours aux technologies de l'information et de la communication représentent plus que des gains d'efficacité ; elles redéfinissent également l'espace argumentatif des possibles, en y introduisant des arguments libérés du contrôle qui s'exerce dans la structure doxique définie par le discours légal, économique et médiatique sur la propriété intellectuelle. Ce contournement, rendu possible par la communication électronique, affecte l'ordre symbolique des actions de lutte pour le logiciel libre, non pas en tissant des liens de solidarité supplémentaires entre communautés d'acteurs mais en autorisant certains arguments à figurer dans l'espace public avec un impact inenvisageable autrement. Dans cette perspective centrée sur la production et les stratégies discursives, qui constituent deux aspects cruciaux des conflits autour de la propriété intellectuelle, Internet apparaît alors comme un dispositif essentiel de la «critique en régime d'impuissance », selon l'expression de Dominique Cardon et Jean-Philippe Heurtin ${ }^{30}$.

${ }^{29}$ M. Diani, « Social Movement Networks, Virtual and Real », op. cit.

30 D. Cardon et J.-P. Heurtin, « La critique en régime d'impuissance. Une lecture des indignations des auditeurs de France-Inter », in B. François, E. Neveu (dir.), Espaces publics mosaïques, Rennes, Presses Universitaires de Rennes, 1999, p. 85-119. 
En plus de leur fonction manifeste de « dispositifs de contre-expertise », servant à opposer les discours à visée dominante et à rendre compte des événements en cours, les sites Internet des activistes du logiciel libre remplissent également une fonction latente de légitimation de leur méthode d'action, en vue de distinguer leur travail de pression du lobbying professionnel engagé par les acteurs privés. La transparence et « l'assiette sociale » des revendications fournissent en effet deux clivages à partir desquels les activistes du logiciel peuvent se revendiquer de défendre publiquement l'intérêt général, par opposition aux acteurs industriels privés dont le lobbying auprès des pouvoirs publics exprime des intérêts particuliers et reste en grande partie dans la sphère privée. Cette division symbolique du travail politique autour de la propriété intellectuelle s'effectue par la mise en ligne des argumentaires, des propositions et des actions des acteurs du logiciel libre, donnant à voir une dimension supplémentaire de l'utilisation des moyens de communication électronique par-delà ses aspects strictement instrumentaux. 